\title{
Palliative Therapy
}

National Cancer Institute

\section{Source}

National Cancer Institute. Palliative Therapy. NCI Thesaurus. Code C15292.

The patient- and family-centered active holistic care of patients with advanced, progressive disease. Essential components of palliative care are: pain and symptom control, communication regarding treatment and alternatives, prognosis, and available services, rehabilitation services, care that addresses treatment and palliative concerns, intellectual, emotional, social, and spiritual needs, terminal care, support in bereavement. The goal of palliative care is an achievement of the best quality of life for patients and their families. 